\title{
Executive Summary: \\ A Grand Challenges-Based Research Agenda for Scholarly Communication and Information Science
}

The "Grand Challenges-Based Research Agenda for Scholarly Communication and Information Science" describes a vision for a more inclusive, open, equitable, and sustainable future for scholarship; characterizes the central technical, organizational, and institutional barriers to this future; describes the areas research needs to advance this future; and identifies targeted "grand challenge" research problems for knowledge generation. These "grand challenges" are fundamental research problems with broad applications, whose solutions are potentially achievable within the next decade.

This executive summary describes the significance of this area, and includes the core recommendations from the report. The full report provides a full discussion of these recommendations, a review of current research, extensive references, and detailed exemplar research questions. The report is available from grandchallenges.pubpub.org .

\section{Significance}

Despite the contested promise of internet technologies for accessibility and democratization, today's scholarly knowledge ecosystem and information sharing environments are plagued by exclusion; inequity; inefficiency; elitism; increasing costs; lack of interoperability; absence of sustainability and/or durability; promotion of commercial rather than public interests; opacity rather than transparency; hoarding rather than sharing; and myriad barriers at individual and institutional levels to access and participation. This report proposes a research agenda guided by the vision of a global information environment that ensures durable, open, equitable, and meaningful global access to knowledge consumption and creation in its many forms.

Such a vision requires the centering of knowledge-producing communities around the world into a global network of partnerships where we all work toward a more inclusive, equitable, trustworthy, and sustainable scholarly knowledge ecosystem—and a durable scholarly record and evidence base. Achieving this vision will require focusing not only on extant systems and processes of knowledge sharing and production, and on recognizing how some participants and forms of knowledge are currently privileged, but also critically evaluating individual and institutional roles and interests that contribute to the current state.

Despite its deep and broad social benefits, science itself remains surprisingly constricted in a number of fundamental aspects: The benefits of science are unevenly distributed. Access to scientific data and scholarly communication, as well as STEM learning materials, has until recently been limited almost exclusively to those inside research or university environments with the ability to pay and fluency in English. Participation in our collective knowledge is limited to a small minority. The vast majority of research that gets into mainstream scholarly publications is conducted in elite university settings in developed countries. Even in those countries, participation in science is heavily skewed by gender, race, class, and language-which affect the construction and evaluation of scientific knowledge. The evidence base is restricted-subjects (people), behaviors, languages, even forms of knowledge, and 
the evidence base in many fields is shifting to new sources. Finally, the algorithms we use to interpret evidence in political and commercial systems embody unexamined bias.

\section{Recommendations for Broad Impact}

The inclusion of people belonging to several communities at once in the creation, dissemination, and use of scholarship is not only ethically imperative, but can strengthen research and scholarship globally, and increase the impacts of scholarship on the world.

In order to promote the broadest impacts of research in this area, in service to the vision of a more inclusive, equitable, and sustainable system of scholarship, we make the following recommendations:

- Recommendation 2-A: We recommend that researchers use rigorous and appropriately transparent methods to consider the broadest possible impact of their work and how that work could be used to improve the inclusiveness and equity of the scholarly knowledge ecosystem.

- Recommendation 2-B: We recommend that research funders include consideration of the impact on the scholarly knowledge ecosystem in their criteria for programs, and that they request applicants to describe the potential for proposed work to increase equity, inclusion, and sustainability. Funders should recognize that some impacts may be far into the future and not easily articulated in the early research stages and give appropriate weight to distant but potentially transformative impacts.

- Recommendation 2-C: We recommend that academic institutions recognize their interdependence and evolve to reflect a systemic approach to reflect the inclusive, open, equitable, and sustainable scholarly knowledge ecosystem essential to our future.

\section{Research Challenges and Barriers}

The report identifies six key challenge to creating a future scholarly knowledge ecosystem which engages people across the world with true opportunities to discover, access, share, and create scholarly knowledge; in which people have agency in their interactions with knowledge systems and control over the information that derives from them; and in which scientific evidence and scholarship are abundant, durable, equitably accessible, and trustworthy.

Participation. Most of the current scholarly knowledge ecosystem contains information produced and controlled by a small part of the world's population. As a consequence, the knowledge, practices, and traditions of many communities are not discoverable, accessible, or preserved. The potential impact of broadening participation in the creation and dissemination of scientific knowledge is substantial. Broadening collection and access to knowledge increasingly depends on the meaningful participation of content creators across the world.

Forms of Knowledge. Current scholarship captures only a small portion of the diverse forms of knowledge. In many communities across the globe, knowledge is based on oral traditions, qualitative and experiential data, and other forms of knowing rarely recognized, valued, or represented in the current scholarly record. A key challenge is to imagine new forms of scholarship that fit new forms of research in order to add new dimensions and perspectives that are broader than the conventional journal article, monograph, and dataset. A second, challenge is to work on ways to make these new genres for scholarship acceptable to research institutions, especially to hiring, promotion, and tenure 
committees. A third challenge is for institutions to provide the infrastructure to support the creation and preservation of these new genres of scholarship, or to pay for scholars to host them elsewhere.

Integrity and Trust. Formal scholarly knowledge generation is limited to small communities, and many members of the public mistrust science — directly, or implicitly. Research is needed in communicating science effectively in increasingly politicized environments. Furthermore, it is increasingly difficult even for scholars to evaluate the weight of the evidence that should be given to claims made in scholarly communications. Current problems are expanding as the scale of scholarly production grows, placing a strain on the mechanisms we have for peer review and quality control.

Durability of Knowledge. The durability of knowledge and scholarship are essential to realizing the full range of scientific discoveries and research, and to establishing the integrity of scholarly knowledge claims. Wiidespread shifts from tangible to digital media create imminent threats to the durability of the scholarly record and scientific evidence base. Further, the digital traces of human behavior have expanded far more rapidly than we can collect, study, and preserve them. Trustworthy scholarship requires that durability is designed into the evolving lifecycle of information creation and use. Moreover, the lack of diversity in the scholarly knowledge ecosystem results in biases not only in what is produced and analyzed, but in what is preserved within the current scholarly knowledge ecosystem.

Individual Agency. Our experiences online are heavily shaped by increasingly complex algorithms, which are often impossible for most participants to fully understand. Further, ubiquitous data collection that gathers information from broad areas of society into academic and commercial research increases the need to maintain privacy, safety, and control, over information. And, as participation in scholarship is broadened, and as community-generated work flows beyond the boundaries of the authoring communities, we will need to honor different community norms on access and use of information.

Incentives for Sustainability. Open scholarship has been a goal for much of the scholarly community for 20-50 years. However, open scholarship is still far from achieving the goals set long ago. Current structures, policies, systems, and norms do not incentivize the behaviors that will lead to the imagined open scholarship future we want.

\section{Recommendations for Research Areas and Programs}

Based on the characterization of the research landscape above, we make the following recommendations:

- Recommendation 3-A: We recommend that funders consider developing future programs and requests for proposals to address the barriers described above.

- Recommendation 3-B: We recommend that researchers in information science and related fields strongly consider selecting problems within a grand-challenge research area as part of their research program.

- Recommendation 3-C: We recommend that reviewers and editors give particular weight to research proposals and discoveries that address these barriers or advance grand-challenge research. 
- Recommendation 3-D: We recommend that the participants in the existing scholarly knowledge ecosystem-including publishers, tool builders, and platform providers-consider how the systems they build can reduce the barriers identified above.

- Recommendation 3-E: We recommend that researchers and stakeholders actively seek out new methodologies, voices, and participation in the design and conduct of research, and also challenge currently accepted ways of conducting, communicating, and evaluating research.

\section{Exemplar Research Questions}

Creating a global scholarly knowledge ecosystem that supports participation, ensures agency, equitable access, trustworthiness, integrity will require exploring a set of interrelated anthropological, behavioral, computational, economic, legal, policy, organizational, sociological, and technological areas. The following questions suggests the range of research areas to be explored:

- What is necessary to develop coherent, comprehensive, and empirically testable theories of the value of scholarly knowledge to society?

- How should the measures of use and utility of scholarly outputs be adapted for different communities of use, disciplines, theories, and cultures?

- How can we develop theories and methods that could reliably summarize the strength of evidence for scholarly knowledge claims?

- How can the mechanisms for promoting trust and trustworthiness be adapted to scholarly contributions by non-professional communities, and applied to non-traditional forms of knowledge?

- What extensions to legal, sociological, organizational, behavioral, and economic theory are necessary in order to create a general, coherent model of a sustainable scholarly knowledge ecosystem that is equitable, trustworthy, and efficient?

- What are the drivers for engagement and participation in scholarly knowledge creation, discovery and curation?

- What are forms of knowledge not represented in the current scholarly knowledge ecosystem?

- What information architecture, semantic analysis, and computational infrastructure is needed to meaningfully link scholarly knowledge across sources and fields of study?

- What parts of the scholarly knowledge ecosystem promote the values of transparency, individual agency, participation, accountability, and fairness?

- What changes in the scholarly ecosystem would enable sustainable intergenerational open access to knowledge?

- What are the most effective modalities for sharing knowledge across different regions and communities, and promoting mutual learning across community boundaries?

The list above is indicative, not comprehensive. The full report outlines the area of research that will need to be addressed in order to overcome the major barriers to a better future for scholarly communication and information science; and provides detailed exemplar research questions. 


\section{Integrating Research, Practice, and Policy}

Many of the opportunities for scholarship that are made possible by rapidly advancing technologies have yet to be fully realized. The social, legal, technical, and organizational systems for disseminating, discovering, reusing, and communicating scholarly information have not kept pace with the technologically induced changes in the scholarly knowledge ecosystem Left to the market, the economics of knowledge in digital form creates both network externalities and reputation effects that are increasingly exploited by rent-seeking monopolies.

Solving this problem requires that institutions coordinate to manage scholarly knowledge-and this requires leadership. Collectively, universities and other institutions must recognize their interdependence and organize as a system to create a scholarly knowledge ecosystem that is not dominated by current market value. Further, research and practice must be dialogue: We need research to guide the design of platforms that are consistent with our values; and platforms that can be instrumented to evaluate these designs, and contribute to our understanding of what we are successfully promoting the objectives we seek.

\section{Recommendations for Integrating Research, Practice, and Policy}

- Recommendation 5-A: We recommend that individual research institutions take public responsibility for leading and coordinating inclusive efforts to address the barriers to more equitable and inclusive systems of scholarship.

- Recommendation 5-B: We recommend that research libraries and archives promote a vision of inclusive and equitable scholarship within their institutions; that they engage in work on legislation and public policy; and that they enlist others in the scholarly community as change-makers.

- Recommendation 5-C: We recommend that those engaged in developing platforms and communities of practices actively seek new voices and participation in their design and use.

- Recommendation 5-D: We recommend that those engaged in research, practice, and advocacy in the area of open and inclusive scholarship should collaborate to develop platforms and interventions that can contribute to our understanding of what is most effective-both directly, and in advancing the broad goals of inclusion, openness, equity, and sustainability. Evaluation of practice should go beyond case studies in their approach, and include replicable methods to support systematic inference.

- Recommendation 5-E: We recommend that stakeholders give priority to resourcing programs that rigorously integrate research and practice, particularly those that systematically contribute to the overall cumulative evidence base for inclusive, equitable, and credible scholarship.

\section{About this Document}

The workshop and paper are supported by a grant from The Andrew W. Mellon Foundation. Contributors to the full report, listed alphabetically: Micah Altman, Chris Bourg, Philip Cohen, G. Sayeed Choudhury, Charles Henry, Sue Kriegsman, Mary Minow, Daisy Selematsela, Anasuya Sengupta, Peter Suber, Ece Turnator, Suzanne Wallen, Trevor Owens, and David Weinberger.

See the full report (grandchallenges.pubpub.org) for a detailed contributor statement and acknowledgements. 